\title{
How Much Atrial Fibrillation is Enough to Warrant Oral Anticoagulation: Management of Subclinical Atrial Fibrillation?
}

Jeff S. Healey

DOI: 10.22374/cjgim.v13iSP1.314

\author{
About the Author \\ Jeff Healey is with the Population Health Research Institute, McMaster University, Hamilton, Ontario Canada. \\ Corresponding Author: Jeff.Healey@phri.ca
}

\begin{abstract}
Stroke due to atrial fibrillation (AF) is common, the cause of significant morbidity and mortality, but is highly preventable with the appropriate use of oral anticoagulants. Recent advances in implantable and wearable electrocardiographic (ECG) technologies now allow continuous monitoring of a patient's heart rhythm for months or years at a time. Cohort studies have shown that using such methods, it is very common to find asymptomatic, short-lasting episodes of subclinical AF. Subclinical AF is also associated with an increased risk of stroke; however, the risk is lower than with traditional, ECG-detected AF and the absolute risk appears to depend on the overall burden of AF. There is currently great uncertainty as to what duration of AF should trigger the use of oral anticoagulation in specific patient groups. Large randomized trials are underway to help clarify this issue; however, in the meantime, researchers and guideline committees have proposed some guidance to assist clinicians.
\end{abstract}

\section{Background}

The use of oral anticoagulants in patients with atrial fibrillation (AF) is one of the most effective strategies to prevent stroke. ${ }^{1,2}$ As our population ages, the risks of both $\mathrm{AF}$ and stroke are increasing. ${ }^{3}$ However; with the advent of safe, and easy-to-use direct oral anticoagulants, ${ }^{2}$ clinicians are increasingly motivated to detect and treat AF. There is optimism in the medical community that screening for AF may be an effective way to reduce the rate of stroke at the population level. ${ }^{4}$

Over the last two decades, there has been substantial development in the tools available to detect $\mathrm{AF}^{4-6}$ In addition to the standard 12-lead electrocardiogram (ECG) Holter monitor and event recorder, there are now a number of devices ranging from handheld or smartphone-based tools to intermittently record a single-lead ECG, ${ }^{5}$ to wearable monitors and patches that can continuously record cardiac activity for several weeks at a time. ${ }^{7,8}$ There are also implantable cardiac monitors, pacemakers and defibrillators that can automatically detect and record AF continuously for many years (Figure 1). ${ }^{6,9,10}$ The use of specific technologies does not yet follow any specific algorithm; however, a more aggressive search for underlying AF using longer-term, continuous monitoring is often employed in high-risk individuals, such as patients who have suffered an embolic stroke. ${ }^{8,9}$ Although there is often a strong clinical desire to treat such individuals with an anticoagulant, it is unclear how much AF must be present to require such therapy. 


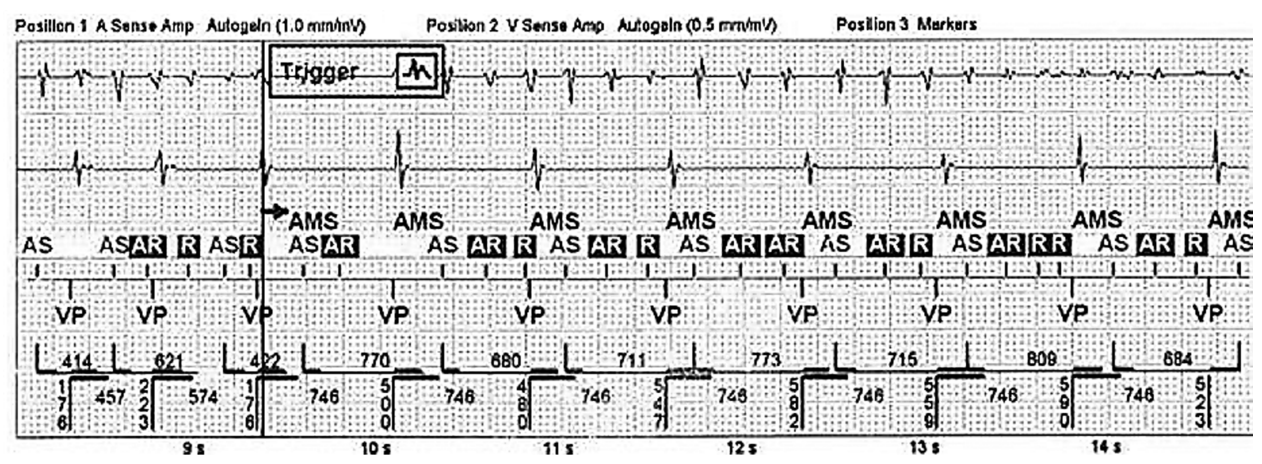

Figure 1. Subclinical atrial fibrillation detected by an implantable pacemaker.

\section{Atrial Fibrillation Defined and Quantified}

Atrial fibrillation can be defined as an abnormal heart rhythm characterized by rapid and irregular beating of the atrium, without electrocardiographic evidence of organized atrial activity and with a duration of at least 30 seconds. The last criterion is similar to that used to distinguish between sustained and non-sustained ventricular tachycardia, and operationally helps to distinguish between AF and non-sustained atrial tachycardia. The latter arrhythmia is extremely common, ${ }^{10,11}$ as even 3 -consecutive premature atrial contractions would be considered an atrial tachycardia. The 30-second minimum duration to define AF thus eliminates any uncertainty around the classification of common, short-lasting, irregular atrial arrhythmia. It has therefore been used as a definition in clinical research to define the presence of AF. ${ }^{8}$ However, it should be recognized that runs of atrial tachycardia lasting only a few seconds, and even frequent premature atrial contractions are associated with an increased risk of stroke; however, this risk is lower than observed for ECG-detected AF and these transient arrhythmias may simply be a marker for longer-lasting $\mathrm{AF}^{12,13}$ It remains unclear if a single, 30-second run of AF detected via long-term monitoring is sufficient to justify lifelong anticoagulation, thus the term "subclinical" AF has been developed to highlight the treatment uncertainty for brief episodes of AF detected in this fashion. ${ }^{6,14}$ As will be shown in this review, the minimum duration of AF necessary to require the use of oral anticoagulation is a subject of great controversial. ${ }^{14}$

Modern recording devices can detect the presence of AF even if it is completely asymptomatic and lasting only seconds at a time. ${ }^{6,10}$ However; these devices can also characterize the frequency, duration and overall burden of AF using a variety of metrics (Figure 2). ${ }^{6,7,15}$ Commonly employed metrics include the duration of the longest $\mathrm{AF}$ episode and the total or average burden of AF (Figure 2). Different studies evaluating the relationship between subclinical AF and stroke have used different metrics, ${ }^{6,15}$ and it is unclear if any metric is superior to any other. Both metrics have potential limitations. Long episodes can be "partitioned" into smaller episodes as a result of under-sensing. ${ }^{16}$ On the other hand, shorter episodes are more likely to represent false-AF detection, ${ }^{16}$ so frequent, short, falsely-detected AF could increase the average detected burden of AF. However; such issues would not have a significant effect on quantification of patients with very long episodes or high burden of subclinical AF; which are the patients for which the association of subclinical AF and stroke is the strongest. ${ }^{15,17}$

While monitoring devices can characterize the amount of AF detected during a particular period of monitoring, one must also consider the implications of the duration of that monitoring interval. The detection of 30 -seconds of AF at the time of a single, 30-second ECG measurement has much different implications than finding a single, 30-second episode of subclinical AF at the time of an annual pacemaker interrogation; the former likely representing a patient with persistent $\mathrm{AF}$, while the latter reflecting

\section{Atrial fibrillation and/or flutter data}

\begin{tabular}{|c|c|}
\hline Duration: & $13 \mathrm{~h} 08 \mathrm{~m} 41 \mathrm{~s}$ \\
\hline Atr. fib. (53.7\%): & $13 \mathrm{~h} 08 \mathrm{~m} 41 \mathrm{~s}$ \\
\hline Atr. flut $(0.0 \%)$ : & $0.00 \mathrm{~s}$ \\
\hline 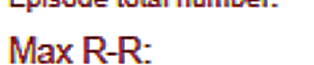 & $1.56 \mathrm{~s}$ \\
\hline Max ventric. rate: & $212 \mathrm{bpm}$ \\
\hline Min Ventric. rate: & 70 bpm \\
\hline Mean Ventric. rate: & $118 \mathrm{bpm}$ \\
\hline Longest eplsode (excl. nolse): & 12h $04 \mathrm{~m} 22 \mathrm{~s}$ \\
\hline & \\
\hline
\end{tabular}

Figure 2. Sample report from a wearable cardiac monitor (CardioSTAT). 
a patient with an extremely low burden of AF. In addition to the characteristics of the AF itself, others have proposed the incorporation of the patient's number of clinical stroke risk factors when assessing the significance of an episode of $\mathrm{AF}^{18}$ The logic is that the minimum duration of $\mathrm{AF}$ that would require treatment is shorter for patients with more stroke risk factors. ${ }^{18}$ While intuitively appealing and consistent with how clinicians manage high-risk individuals, such as patients who have suffered an embolic stroke, ${ }^{14}$ none of these methods has been clearly shown to identify which individuals with short-lasting subclinical AF require anticoagulation. However; a great deal of analyses has been conducted to increase our understanding of this issue, and several large, intervention studies are now underway. ${ }^{19,20}$

The association between $\mathrm{AF}$ and stroke was established by large, epidemiological studies, which ascertained AF by conducting a standard electrocardiogram one or twice per year ${ }^{21,22}$. These studies found that the risk of stroke was increased 4 - to 5 -fold in patients with AF detected in this manner, ${ }^{21,22}$ and that the absolute risk of stroke is greater among patients with additional stroke risk factors. ${ }^{13}$ However, even with ECG-detected AF, recent research suggests an association between the burden of $\mathrm{AF}$ and the risk of stroke. In a large cohort of patients with established AF who were receiving only anti-platelet therapy, it was demonstrated that after correction for other stroke risk factors, patients with permanent AF, had about double the absolute of stroke as patients with paroxysmal AF (i.e., not present on all ECGs during the study), with patients characterized as persistent AF having an intermediate risk. ${ }^{23}$ However, in this population of individuals who all had additional stroke risk factors, patients with paroxysmal AF still had an annual risk of stroke well-above the cutoff for using oral anticoagulation. ${ }^{14}$ Thus, although the characterization of clinical AF type does not impact the decision to anticoagulate, AF type does have an impact on a patient's absolute risk of stroke. This association between AF burden and stroke risk is even more apparent in patients with shorter-lasting episodes of subclinical AF.

\section{Subclinical Atrial Fibrillation: Prevalence and Association with Stroke}

In the 1990s, pacemakers were introduced that allowed detection and characterization of atrial arrhythmias. ${ }^{24}$ However, the detection of subclinical AF typically did not result in a decision to anticoagulate patients, given the uncertainty regarding the risk of stroke in patients with only subclinical AF, and the limitations of warfarin, the only indicated medication at that time. ${ }^{25}$ Two large prospective cohort studies were conducted to address the former issue: the TRENDS study ${ }^{15}$ and the ASSERT study. ${ }^{26}$ The TRENDS study defined patients as having subclinical AF if AF lasting at least 5 minutes was detected, and demonstrated a borderline-significant 2.2-fold increase risk of stroke among patients with subclinical AF, whose maximum average daily burden in any 30-day periods was over 5.5 hours. ${ }^{15}$ This study did not find any increase in the risk of stroke among patients with lesser amounts of AF. The ASSERT trial took a different approach, and characterized patients as having subclinical AF if any episode lasting 6 or more minutes was detected, and then categorized patients as having (vs. not having) subclinical AF 3 months after study enrollment. ASSERT detected a statistically significant increase in stroke risk of 2.5 -fold among patients with subclinical AF. However, the absolute risk of stroke among these patients was only $1.69 \%$ per year; much lower than was predicted based on patients with clinically detected AF and similar clinical stroke risk factors. ${ }^{6}$ A subsequent, time-dependent analysis of the ASSERT data was conducted, which demonstrated that most of the increased risk of stroke among patients with subclinical AF was concentrated among patients with discrete episodes lasting at least 24 continuous hours. ${ }^{17}$ These patients had about a 5 -fold increase in stroke risk, with absolute stroke risk of approximately $5 \%$ per year; quite similar to patients with clinically detected AF. ${ }^{13,17}$ In patients whose longest episode of subclinical AF was less than 24 hours, no increased risk of stroke was observed ${ }^{13}$. Thus, although both TRENDS and ASSERT show an association between subclinical AF as brief as 5-6 minutes in duration and stroke; both studies also suggest that this risk is confined to patients with longer episodes or higher burden of AF.

The TRENDS and ASSERT trials also shed light on the precise temporal relationship between subclinical AF and stroke. ${ }^{26,27}$ As all patients in these studies had continuous ECG monitoring throughout follow-up, analysis of pacemaker logs could tell how much AF was present in patients who suffered stroke before the stroke occurred. The studies had consistent findings that only $15-20 \%$ of patients who suffered stroke had any history of AF in the month prior to the stroke. ${ }^{26,27}$ The absence of long-lasting episodes with close temporal association to stroke is at odds with the classical teaching that thrombus develops if AF lasts more than 24 hours and subsequently embolizes to cause stroke. This raised the possibility that subclinical AF may not only be acting as a direct causal factor for stroke in these patients, but may also behave like a vascular risk marker. This observation is in keeping with the association between frequent PACs and very brief (20 beats) runs of atrial tachycardia and stroke seen in another cohort study. ${ }^{12}$ Finally, a blinded adjudication of all strokes in the ASSERT trial demonstrated that although embolic-appearing strokes were more common in patients with subclinical AF, a similar number of patients suffered lacunar stroke (which is not felt to be embolic) and smaller numbers suffered stroke from other mechanisms, such as carotid atherosclerosis. ${ }^{28}$ As patients who develop subclinical AF are older and typically have other cardiovascular conditions, it should not be surprising that stroke 
may occur due to a variety of mechanism, sometimes in the same individual. This makes the relationship between subclinical AF and stroke complex in these individuals, with subclinical AF acting in a causal way sometimes, and as a vascular risk marker at others.

\section{Prevalence and Implications of Subclinical Atrial Fibrillation}

While cohort studies have shown that subclinical AF is present in $30-50 \%$ of patients with pacemakers and defibrillators, ${ }^{6,15}$ it is not was not initially clear if these patients were at a particularly high risk of subclinical AF, or at a similar risk to other older patients without pacemakers but with cardiovascular risk factors. Similarly, long-term monitoring detected subclinical AF in 15-30\% of individuals who suffered cryptogenic stroke. ${ }^{8,9}$ As neither of these landmark studies included a control group, it was unclear if these patients were at greater risk of subclinical AF, or at similar risk as individuals of the same age with similar comorbidities. ${ }^{89}$ Thus, several studies were undertaken (Table 1) to define the prevalence of subclinical AF in a more general population of older individuals with cardiovascular conditions. ${ }^{10,29,30}$ These studies were quite consistent in finding a very high background prevalence of subclinical AF, which was at least as high as in patients with pacemaker or following cryptogenic stroke, (Table 1). ${ }^{6,9}$

Finding subclinical AF of at least 5 minutes duration in $25-35 \%$ of older individuals with cardiovascular conditions has implies that this condition is not exceptional, and that upon detecting such episodes, clinicians should not automatically assume that anticoagulation is required. This was highlighted with the recent results of the NAVIGATE-ESUS trial, which evaluated the routine use of oral anticoagulation in patients following an embolic stroke of undetermined source. ${ }^{31}$ Despite the fact that one would expect to detect subclinical AF in more than $30 \%$ of such patients, ${ }^{9}$ routine treatment with rivaroxaban did not prevent stroke.31 However, the COMPASS trial demonstrated that aspirin plus a low dose of rivaroxaban was associated with a $42 \%$ reduction in stroke compared to aspirin alone among individuals with cardiac disease but without manifest clinical $\mathrm{AF}^{32}$ Thus, it remains unclear if any population with a high prevalence of subclinical AF benefits from empiric antithrombotic therapy to prevent stroke, or if monitoring for subclinical AF and treatment if $\mathrm{AF}$ is detected should be the preferred strategy. Randomized intervention studies are now ongoing to address this question. ${ }^{20,33}$

Two large randomized trials are underway in patients with pacemakers, implanted defibrillators or cardiac monitors. The ARTESiA trial will enroll patients with subclinical AF of between 6 minutes and 24 hours duration and randomize to apixaban or aspirin. ${ }^{34}$ The NOAH-AFNET- 6 will enroll patients with subclinical $\mathrm{AF}$ of at least 6 minutes, and both trials will require that patients have additional stroke risk factors. ${ }^{19,20}$ Both trials are well underway with enrollment, and should present results in the next 2-4 years. Both trials will also examine the effect of anticoagulant treatment for patients with subclinical AF duration or burden above versus below the median value of the cohort; to evaluate if there is a differential treatment effect for individuals with longer episodes.

\section{Clinical Implications}

Subclinical AF is very common in older individuals with cardiovascular and stroke risk factors. Although it is associated with an increased risk of stroke, this risk is lower than with traditional, clinical AF and may be dependent on the burden of arrhythmia. Pending the results of ongoing studies, it seems prudent to offer oral anticoagulation to individuals with subclinical AF of greater than 24 hours duration that is detected by long-term continuous monitoring (i.e., pacemaker, defibrillator or implanted cardiac monitor). For episodes less than 6 minutes duration there is no clear association with stroke risk, while for episodes of between 6 minutes and 24 hours, this is association is unclear, but may be present for patients with greater AF burden.

Table 1. Cohort Studies to Define Prevalence of Subclinical AF

\begin{tabular}{|c|c|c|c|}
\hline Study & Sample Size & Inclusion & Rate of AF Detection \\
\hline ASSERT-II ${ }^{10}$ & 250 & $\begin{array}{l}\text { Age }>65, \text { AND } \\
\text { CHADS-VASc } \geq 2 \text {, or OSA, or BMl }>30 \text {; AND } \\
L A>58 \mathrm{~mL} \text {, or NT-ProBNP }>290 \mathrm{pg} / \mathrm{mL}\end{array}$ & $\begin{array}{l}\geq 5 \mathrm{~min} \\
34.4 \% \text { at one year }\end{array}$ \\
\hline REVEAL-AF ${ }^{29}$ & 450 & $\begin{array}{l}\text { Age } \geq 18 \\
\text { CHADS } \geq 3 \text {, or CKD/COPD/OSA/CAD }\end{array}$ & $29.3 \%$ at 18 months \\
\hline PREDATE-AF ${ }^{30}$ & 245 & $\begin{array}{l}\text { Age }>18, \text { AND } \\
\text { CHADS-VASC } \geq 2\end{array}$ & $\begin{array}{l}\geq 6 \min \\
22.4 \% \text { at } 451 \text { days }\end{array}$ \\
\hline DANISH LOOP ${ }^{35}$ & 6000 & $\begin{array}{l}\text { Age }>70 \\
\text { One of HTN, DM, HF or stroke }\end{array}$ & Pending \\
\hline
\end{tabular}

$\mathrm{AF}=$ atrial fibrillation; $\mathrm{BMI}=$ body mass index; $\mathrm{CAD}=$ coronary artery disease; $\mathrm{CKD}=$ chronic kidney disease; $\mathrm{COPD}=$ chronic obstructive pulmonary disorder; $\mathrm{DM}=$ diabetes milletus; $\mathrm{HF}=$ heart failure; HTN = hypertension. 
For patients undergoing monitoring of shorter duration, the corresponding amount of AF that should trigger treatment is unknown, but presumably proportionally shorter. A metric such as average daily burden of AF might be useful to make such evaluation. However, most individuals in whom subclinical AF is detected by a single-time-point, intermittent method (like a 6-second ECG or 30-second handheld recording) should receive anticoagulation according to practice guidelines. ${ }^{14}$ Finally, in certain high-risk populations, such as those with a recent embolic stroke of unknown source, it may be reasonable to have a much lower threshold to treat subclinical AF, even if trial data are lacking.

\section{References}

1. Hart RG, Pearce LA, Aguilar MI. Meta-analysis: antithrombotic therapy to prevent stroke in patients who have nonvalvular atrial fibrillation. Ann Intern Med 2007;146(12):857-67.

2. Ruff CT, Giugliano RP, Braunwald E, et al. Comparison of the efficacy and safety of new oral anticoagulants with warfarin in patients with atrial fibrillation: a meta-analysis of randomised trials. Lancet (London, England). 2014;383(9921):955-62.

3. Chugh SS, Havmoeller R, Narayanan K, et al. Worldwide epidemiology of atrial fibrillation: a Global Burden of Disease 2010 Study. Circulation 2014;129(8):837-47.

4. Freedman B, Camm J, Calkins H, et al. Screening for Atrial Fibrillation: A Report of the AF-SCREEN International Collaboration. Circulation 2017;135(19):1851-67.

5. Lau JK, Lowres N, Neubeck L, et al. iPhone ECG application for community screening to detect silent atrial fibrillation: a novel technology to prevent stroke. Int J Cardiol 2013;165(1):193-94.

6. Healey JS, Connolly SJ, Gold MR, et al. Subclinical atrial fibrillation and the risk of stroke. N Engle J Med 2012;366(2):120-29.

7. Turakhia MP, Ullal AJ, Hoang DD, et al. Feasibility of extended ambulatory electrocardiogram monitoring to identify silent atrial fibrillation in highrisk patients: the Screening Study for Undiagnosed Atrial Fibrillation (STUDY-AF). Clin Cardiol 2015;38(5):285-92.

8. Gladstone DJ, Spring M, Dorian P, et al. Atrial fibrillation in patients with cryptogenic stroke. N Engl J Med 2014;370(26):2467-77.

9. Sanna T, Diener HC, Passman RS, et al. Cryptogenic stroke and underlying atrial fibrillation. N Engl J Med 2014;370(26):2478-86.

10. Healey JS, Alings M, Ha A, et al. Subclinical atrial fibrillation in older patients. Circulation 2017;136(14):1276-83.

11. Gladstone DJ, Dorian P, Spring M, et al. Atrial premature beats predict atrial fibrillation in cryptogenic stroke: results from the EMBRACE trial. Stroke 2015;46(4):936-41.

12. Binici Z, Intzilakis T, Nielsen OW, Kober L, Sajadieh A. Excessive supraventricular ectopic activity and increased risk of atrial fibrillation and stroke. Circulation 2010;121(17):1904-11.

13. Gage BF, Waterman AD, Shannon W, Boechler M, Rich MW, Radford MJ. Validation of clinical classification schemes for predicting stroke: results from the National Registry of Atrial Fibrillation. JAMA 2001;285(22):2864-70.

14. Verma A, Cairns JA, Mitchell LB, et al. 2014 focused update of the Canadian Cardiovascular Society Guidelines for the management of atrial fibrillation. Can J Cardiol 2014;30(10):1114-30.

15. Glotzer TV, Daoud EG, Wyse DG, et al. The relationship between daily atrial tachyarrhythmia burden from implantable device diagnostics and stroke risk: the TRENDS study. Circ Arrhythm Electrophysiol 2009;2(5):474-80.

16. Kaufman ES, Israel CW, Nair GM, et al. Positive predictive value of devicedetected atrial high-rate episodes at different rates and durations: an analysis from ASSERT. Heart Rhythm 2012;9(8):1241-46.
17. Van Gelder IC, Healey JS, Crijns HJ, et al. Duration of device-detected subclinical atrial fibrillation and occurrence of stroke in ASSERT. Eur Heart J 2017.

18. Botto GL, Padeletti L, Santini M, et al. Presence and duration of atrial fibrillation detected by continuous monitoring: crucial implications for the risk of thromboembolic events. J Cardiovasc Electrophysiol 2009;20(3):241-48.

19. Lopes RD, Alings M, Connolly SJ, et al. Rationale and design of the Apixaban for the Reduction of Thrombo-Embolism in Patients With DeviceDetected Sub-Clinical Atrial Fibrillation (ARTESiA) trial. Am Heart J 2017;189:137-45.

20. Kirchhof P, Blank BF, Calvert M, et al. Probing oral anticoagulation in patients with atrial high rate episodes: Rationale and design of the Nonvitamin $\mathrm{K}$ antagonist Oral anticoagulants in patients with Atrial High rate episodes (NOAH-AFNET 6) trial. Am Heart J 2017;190:12-18.

21. Wolf PA, Abbott RD, Kannel WB. Atrial fibrillation as an independent risk factor for stroke: the Framingham Study. Stroke 1991;22(8):983-88.

22. Wolf PA, Kannel WB, McGee DL, Meeks SL, Bharucha NE, McNamara PM. Duration of atrial fibrillation and imminence of stroke: the Framingham study. Stroke 1983;14(5):664-67.

23. Vanassche T, Lauw MN, Eikelboom JW, et al. Risk of ischaemic stroke according to pattern of atrial fibrillation: analysis of 6563 aspirin-treated patients in ACTIVE-A and AVERROES. Eur Heart J 2015;36(5):281-87a.

24. Glotzer TV, Hellkamp AS, Zimmerman J, et al. Atrial high rate episodes detected by pacemaker diagnostics predict death and stroke: report of the Atrial Diagnostics Ancillary Study of the MOde Selection Trial (MOST). Circulation 2003;107(12):1614-19.

25. Healey JS, Martin JL, Duncan A, et al. Pacemaker-detected atrial fibrillation in patients with pacemakers: prevalence, predictors, and current use of oral anticoagulation. Can J Cardiol 2013;29(2):224-28.

26. Daoud EG, Glotzer TV, Wyse DG, et al. Temporal relationship of atrial tachyarrhythmias, cerebrovascular events, and systemic emboli based on stored device data: a subgroup analysis of TRENDS. Heart Rhythm 2011;8(9):1416-23.

27. Brambatti M, Connolly SJ, Gold MR, et al. Temporal relationship between subclinical atrial fibrillation and embolic events. Circulation 2014;129(21):2094-99.

28. Perera KS, Sharma M, Connolly SJ, et al. Stroke type and severity in patients with subclinical atrial fibrillation: An analysis from the Asymptomatic Atrial Fibrillation and Stroke Evaluation in Pacemaker Patients and the Atrial Fibrillation Reduction Atrial Pacing Trial (ASSERT). Am Heart J 2018.

29. Reiffel JA, Verma A, Kowey PR, et al. Incidence of Previously Undiagnosed Atrial Fibrillation Using Insertable Cardiac Monitors in a High-Risk Population: The REVEAL AF Study. JAMA Cardiol 2017;2(10):1120-27.

30. Nasir JM, Pomeroy W, Marler A, et al. Predicting Determinants of Atrial Fibrillation or Flutter for Therapy Elucidation in Patients at Risk for Thromboembolic Events (PREDATE AF) Study. Heart Rhythm 2017;14(7):955-61.

31. Hart RG, Sharma M, Mundl H, et al. Rivaroxaban for Stroke Prevention after Embolic Stroke of Undetermined Source. N Engl J Med 2018;378(23):2191-201.

32. Eikelboom JW, Connolly SJ, Bosch J, et al. Rivaroxaban with or without Aspirin in Stable Cardiovascular Disease. N Engl J Med 2017;377(14):1319-30.

33. Lopes RD. Rationale and design of the Apixaban for the Reduction of Thrombo-Embolism in Patients With Device-Detected Sub-Clinical Atrial Fibrillation (ARTESiA) trial. Am Heart J 2017 189:137-45. doi: 10.1016/j. ahj.2017.04.008. Epub 2017 Apr 24..

34. Camm AJ, Amarenco P, Haas S, et al. XANTUS: a real-world, prospective, observational study of patients treated with rivaroxaban for stroke prevention in atrial fibrillation. Eur Heart J 2016;37(14):1145-53.

35. Diederichsen SZ, Haugan KJ, Hojberg S, et al. Complications after implantation of a new-generation insertable cardiac monitor: Results from the LOOP study. In J Cardiol 2017;241:229-34. 